\title{
Radiomic signature
}

Ashwini Kalshetty

Potential competing interests: The author(s) declared that no potential competing interests exist.

a unique set of radiomic features which serves as an imaging bio-marker characteristic of a clinical condition or process. 